\title{
THE REALIZATION OF INVERSE SYSTEM FOR CIRCUITS CONTAINING NULLORS WITH APPLICATIONS IN CHAOS SYNCHRONIZATION
}

\author{
ADRIAN LEUCIUC* \\ Department of Electronics and Telecommunications, Technical University of lasi, P.0. Box 877, Iasi 6600, Romania
}

\begin{abstract}
SUMMARY
A very simple method for synthesizing the inverse system of a non-linear non-autonomous circuit containing nullors is proposed. The main application of the procedure is the synchronization of chaos by the inverse system approach. This is illustrated with two examples: the synchronization of a Duffing circuit and a communication scheme by direct chaotic modulation using Chua's circuit. (C) 1998 John Wiley \& Sons, Ltd.
\end{abstract}

KEY WORDS: non-linear circuit; synchronization; chaos; inverse system; Duffing circuit

\section{INTRODUCTION}

Since 1990 when Pecora and Carroll ${ }^{1}$ published their results on chaotic synchronization of two systems this problem has become one of the most popular in the scientific world. There are three basic methods to synchronize chaotic systems. ${ }^{2}$

1. by decomposition into subsystems; ${ }^{3}$

2. by linear feedback; ${ }^{4-6}$

3. by the inverse system. ${ }^{7,8}$

The last method can be used for synchronizing non-autonomous systems. Basically, the inverse system approach is depicted in Figure 1, where the system 1 is the master producing the output $y(t)$ when excited with $u(t)$. The chaotic signal $y(t)$ drives the slave system 2 which is the inverse of system 1 and, thus, if the initial conditions are identical, its output will follow the input of the master system. In practice, since we do not have control on initial conditions, the inverse system may or may not synchronize. Synchronization will occur if all conditional Lyapunov exponents (CLE) of the slave system are negative. ${ }^{9}$ The simplest way to realize the inverse system is that of driving a non-linear one port by a voltage source, transmitting the current through the one port and driving with this signal and identical circuit. ${ }^{2}$ If the receiver is open circuit asymptotically stable, after some time the voltage across the slave circuit will follow the input of the master. In this paper another procedure for realizing the inverse systems for circuits containing nullors is proposed. In Section 2 the linear case is investigated and a rigorous proof for the implementation of the inverse transfer function is given. The non-linear systems are treated in Section 3 and two examples of chaos synchronization in the Duffing and Chua circuits are presented. In Section 4 a procedure to generate nullor-based circuits in order to use the previous obtained results for any chaotic circuit is proposed.

* Correspondence to: A. Leuciuc, Department of Electronics and Telecommunications, Technical University of Iasi, P.O. Box 877, Iasi, 6600, Romania. E-mail: aleuciuc@tuiasi.ro

CCC 0098-9886/98/010001-12\$17.50

Received 14 March 1996

(C) 1998 John Wiley \& Sons, Ltd. Revised 29 April 1997 


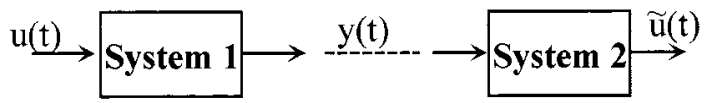

Figure 1. Chaos synchronization by the inverse system approach

\section{INVERSE TRANSFER FUNCTION REALIZATION IN LINEAR CIRCUITS WITH NULLORS}

One of the most popular devices used in circuit synthesis is the operational amplifier. At low frequencies this device can be represented by an ideal op-amp model, which is equivalent with a nullor, the well-known two-port circuit element. Besides op-amp several other devices can be modelled by nullors: the operational mirrored amplifier, ${ }^{10}$ the second-generation current conveyor. ${ }^{11}$ In the following a theorem giving a method for generating the inverse transfer function for a linear circuit is introduced.

Theorem 1. Let us consider a linear-time-invariant non-autonomous circuit containing at least one nullor and excited by a voltage source (Figure 2). Suppose the system has a transfer function $F(s)$ with respect to the output the voltage across the norator $\left(V_{3}\right)$, respectively, $G(s)$ if the output is the current through the norator $\left(I_{3}\right)$. Replacing the input voltage source with the norator and, depending on the output variable, the norator of Figure 2 with a voltage, respectively a current source, as is shown in Figure 3, the new circuits have transfer functions equal to $F^{-1}(s)$ and $G^{-1}(s)$ with respect to the voltage at port 1.

Proof. Considering the admittance matrix description of the linear three port in Figure 2 one has

$$
\begin{aligned}
& I_{1}=y_{11} V_{1}+y_{12} V_{2}+y_{13} V_{3} \\
& I_{2}=y_{21} V_{1}+y_{22} V_{2}+y_{23} V_{3} \\
& I_{3}=y_{31} V_{1}+y_{32} V_{2}+y_{33} V_{3}
\end{aligned}
$$

The nullator forces $I_{2}=0$ and $V_{2}=0$. Thus, the voltage transfer function $F(s)$ and the transfer admittance $G(s)$ are

$$
\begin{aligned}
& F(s)=\frac{V_{3}}{V_{1}}=-\frac{y_{21}}{y_{23}} \\
& G(s)=\frac{I_{3}}{V_{1}}=\frac{y_{31} y_{23}-y_{33} y_{21}}{y_{23}}
\end{aligned}
$$

For the circuits of Figures 3(a) and 3(b) similar equations (1) hold

$$
\begin{aligned}
& \tilde{I}_{1}=y_{11} \tilde{V}_{1}+y_{12} \tilde{V}_{2}+y_{13} \tilde{V}_{3} \\
& \tilde{I}_{2}=y_{21} \tilde{V}_{1}+y_{22} \tilde{V}_{2}+y_{23} \tilde{V}_{3} \\
& \tilde{I}_{3}=y_{31} \tilde{V}_{1}+y_{32} \tilde{V}_{2}+y_{33} \tilde{V}_{3}
\end{aligned}
$$

The nullator sets $\tilde{I}_{2}=0, \tilde{V}_{2}=0$ and a simple analysis yields

$$
\begin{aligned}
& \tilde{F}(s)=\frac{\tilde{V}_{1}}{\tilde{V}_{3}}=-\frac{y_{23}}{y_{21}}=F^{-1}(s) \\
& \tilde{G}(s)=\frac{\tilde{V}_{1}}{\tilde{I}_{3}}=\frac{y_{23}}{y_{31} y_{23}-y_{33} y_{21}}=G^{-1}(s)
\end{aligned}
$$




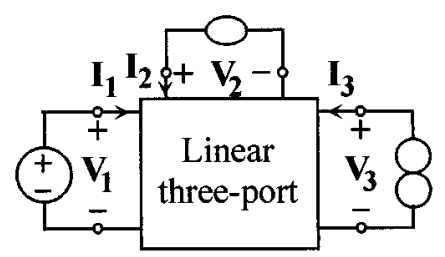

Figure 2. Linear master circuit containing at least one nullor and driven by a voltage source

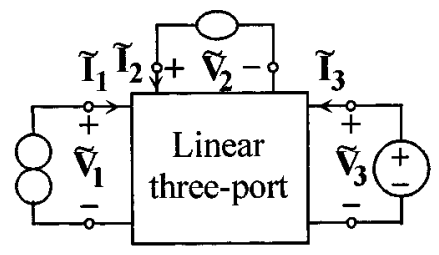

(a)

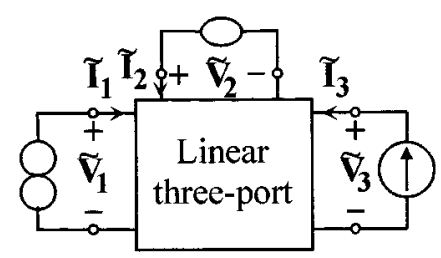

(b)

Figure 3. Realization of inverse transfer function systems for linear circuit containing nullors: (a) inverse system in the case of voltage output; (b) inverse system in the case of current output

Remark. Theorem 1 provides a method for synthesizing a circuit possessing inverse transfer function of a given master linear circuit. However, it does not follow that the configurations of Figure 3 are the inverse of the one depicted in Figure 2 in the sense that if $\tilde{V}_{3}=V_{3}$ or $\tilde{I}_{3}=I_{3}$ then all the voltages and currents of these circuits will follow after some time the variables from the master. The necessary and sufficient condition for this to occur is that the slave be stable, that is the transfer function of the master circuit be minimum phase. The linearity of the transmitter and receiver ensures in this case the synchronization of the slave system for any initial state. In the case of current-driven circuit similar results can be derived by duality.

\section{THE NON-LINEAR CASE}

Based on the results obtained in the case of linear circuits, the same procedure described previously can be used to generate the inverse system for non-linear circuits. The corresponding structures of the transmitter and receiver in the case of voltage-driven master circuit and voltage output variable are depicted in Figures 4 and 5, respectively. Similar configurations can be easily obtained in the cases of exciting the master with a current source and/or considering current type output.

Theorem 2. If for any inputs $u$ and $y$ the non-linear dynamical systems of Figures 4 and 5, respectively, have unique bounded solutions, then they are inverse to each other, that is for every input signal $u$ and every initial state $\mathbf{x}(0)$ of the master there is an initial state $\tilde{\mathbf{x}}(0)$ of the slave such that $\tilde{u}(\tilde{\mathbf{x}}(0), y(\mathbf{x}(0), u))=u$. 


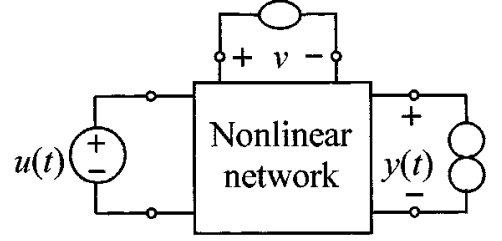

Figure 4. Non-linear non-autonomous system containing at least one nullor

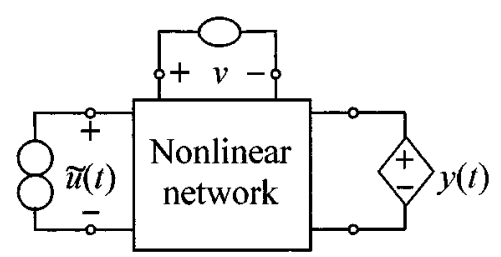

Figure 5. The inverse system of the circuit of Fig. 4

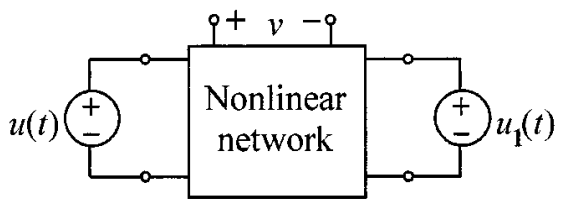

Figure 6. Two inputs non-autonomous circuit used in the proof of Theorem 2

Proof. Let us consider the non-autonomous non-linear circuit driven by two independent voltage sources $u$ and $u_{1}$ of Figure 6 . Considering as output the voltage $v$, the following state and output equations can be written:

$$
\begin{aligned}
\dot{\mathbf{x}} & =\mathbf{f}\left(\mathbf{x}, u, u_{1}\right) \\
v & =g\left(\mathbf{x}, u, u_{1}\right)
\end{aligned}
$$

where $\mathbf{f}: \mathbf{R}^{n} \times D \times D_{1} \rightarrow \mathbf{R}^{n}, g: \mathbf{R}^{n} \times D \times D_{1} \rightarrow \mathbf{R}, D$ and $D_{1}$ being the sets of admissible input signals $u$ and $u_{1}$.

If the circuit of Figure 6 is augmented with a nullator at the top port, a supplementary constraint $v=0$ is imposed and this leads to a non-physical situation. To eliminate this problem, one of the two independent sources must be replaced by a norator which behaves like a voltage source whose value is set by the constraints imposed by the nullator. Thus, the two configurations of Figures 4 and 5 are obtained. Since the topology of the circuits remains unchanged they will be described by the following equations.

for the circuit of Figure 4:

$$
\begin{aligned}
\dot{\mathbf{x}} & =\mathbf{f}(\mathbf{x}, u, y) \\
0 & =g(\mathbf{x}, u, y)
\end{aligned}
$$

for the circuit of Figure 5:

$$
\begin{aligned}
& \dot{\tilde{\mathbf{x}}}=\mathbf{f}(\tilde{\mathbf{x}}, \tilde{u}, y) \\
& 0=g(\tilde{\mathbf{x}}, \tilde{u}, y)
\end{aligned}
$$

If the two circuits have unique behaviour then the equation $g(\mathbf{x}, u, y)=0$ has unique solution $y=h(\mathbf{x}, u)$ and the equation $g(\tilde{\mathbf{x}}, \tilde{u}, y)=0$ has also unique solution $\tilde{u}=h^{-1}(\tilde{\mathbf{x}}, y)$ for every $\mathbf{x}, \tilde{\mathbf{x}} \in \mathbf{R}^{n}$. Thus, the state and output equations describing the dynamics of the circuits of Figures 4 and 5 are

$$
\begin{aligned}
\dot{\mathbf{x}} & =\mathbf{f}(\mathbf{x}, u, h(\mathbf{x}, u)) \\
y & =h(\mathbf{x}, u)
\end{aligned}
$$


respectively,

$$
\begin{aligned}
\dot{\tilde{\mathbf{x}}} & =\mathbf{f}\left(\tilde{\mathbf{x}}, h^{-1}(\tilde{\mathbf{x}}, y), y\right) \\
\tilde{u} & =h^{-1}(\tilde{\mathbf{x}}, y)
\end{aligned}
$$

It is obvious that if $y$ is a bounded solution of (10) and the slave has also bounded solutions, then for $\mathbf{x}(0)=\tilde{\mathbf{x}}(0), \tilde{u}=u$ is a solution for $(11)$.

One has to remark that the equation $g(\mathbf{x}, u, y)=0$ has always unique solution $y=h(\mathbf{x}, u)$ for every $\mathbf{x} \in \mathbf{R}^{n}$ since the master circuit of Figure 4 is chaotic with well-defined behaviour. The inverse system exists if the function $h(\mathbf{x}, u)$ is invertible with respect to $u$ on the entire state space. The resulting circuit has equal order with the master.

If the relative degree ${ }^{7}$ of the master $r \neq 0$, then $g(\mathbf{x}, u, y)=g(\mathbf{x}, y)$ and the output depends only on the state variables $y=h(\mathbf{x})$. In this case the inverse system, if exists, is of lower order than the original one. A necessary condition for the existence of the inverse system is the invertibility of the function $h(\mathbf{x})$ with respect to one of the state variables $x_{k}$ for every $\left(x_{1}, \ldots, x_{k-1}, x_{k+1}, \ldots, x_{n}\right) \in \mathbf{R}^{n-1}$. The proof can be derived in a similar manner as in Theorem 2 .

The inverse system synchronizes with the master, $\tilde{u} \rightarrow u$ as $t \rightarrow \infty$ for any initial states $\mathbf{x}(0)$ and $\tilde{\mathbf{x}}(0)$, if all its conditional Lyapunov exponents are negative, i.e. it has unique asymptotic behaviour. If the state equation of the inverse system is linear, the conditional Lyapunov exponents are equal to its eigenvalues. This feature will be used in the following examples to prove the synchronization. In the case $r \neq 0$ the recovered signal $\tilde{u}$ depends on the derivatives of the transmitted signal $y$ up to $n-r$ order. The synchronization is in this case very sensitive to noise and variation of parameters.

In a practical realization it is rather possible that the voltage across the norator or the current through it not to fulfill the conditions ensuring the existence of inverse system and its synchronization. However, if any other variable, voltage or current, from the master circuit satisfies these requirements, it is always possible to build the inverse system using an extra nullor as it will be pointed out in Section 4.

Remark: The $\mathrm{V} \rightarrow \mathrm{I} \rightarrow \mathrm{V}$ method for realizing system inversion using an op-amp ${ }^{7,8}$ is a particular case of the proposed procedure.

\subsection{Example 1: Duffing circuit}

The Duffing equation is one of the most studied non-linear non-autonomous system exhibiting various dynamics, including chaos and bifurcations:

$$
\begin{gathered}
\ddot{x}_{1}+a \dot{x}_{1}+f\left(x_{1}\right)=q \cos \omega t \\
f\left(x_{1}\right)=p x_{1}+x_{1}^{3}
\end{gathered}
$$

One of the possible active configurations described by (12) and obtained on the basis of Antoniou's General Impedance Converter ${ }^{12,13}$ is presented in Figure 7. Since this structure contains two op-amps operating in the linear region, one can use the procedure described above to build a slave system which synchronizes with the master when the voltage from the output of one op-amp is transmitted. Denoting by $x_{1}$ the voltage across the capacitor $C_{2}$ and by $x_{2}$ the voltage across the capacitor $C_{4}$ and considering ideal op-amps the following state equation is obtained for the circuit of Figure 7:

$$
\begin{aligned}
& \dot{x}_{1}=-a x_{1}+x_{2} \\
& \dot{x}_{2}=-f\left(x_{1}\right)+u(t)
\end{aligned}
$$


equivalent with (12) for $u(t)=q \cos \omega t$. The voltages $y_{1}$ and $y_{2}$ at the outputs of the two op-amps are, respectively,

$$
\begin{aligned}
& y_{1}=-f\left(x_{1}\right)+x_{2}+u \\
& y_{2}=-x_{1}-f\left(x_{1}\right)+u
\end{aligned}
$$

The slave configurations corresponding to the two transmitted signals are depicted in Figure 8 and are described by the following sets of equations.

for the circuit of Figure 8(a):

$$
\begin{aligned}
\dot{\tilde{x}}_{1} & =-a \tilde{x}_{1}+\tilde{x}_{2} \\
\dot{\tilde{x}}_{2} & =-\tilde{x}_{2}+y_{1} \\
\tilde{u} & =f\left(\tilde{x}_{1}\right)-\tilde{x}_{2}+y_{1}
\end{aligned}
$$

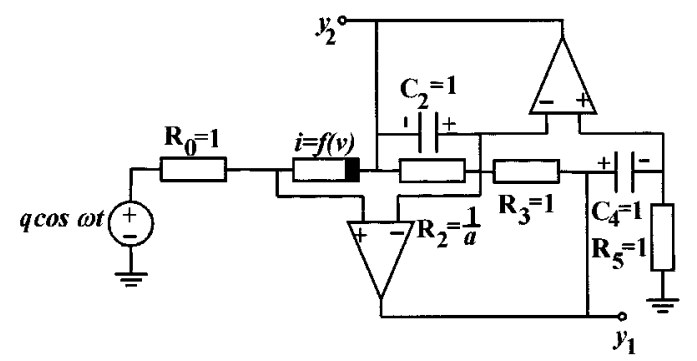

Figure 7. Active implementation of Duffing equation using Antoniou's GIC

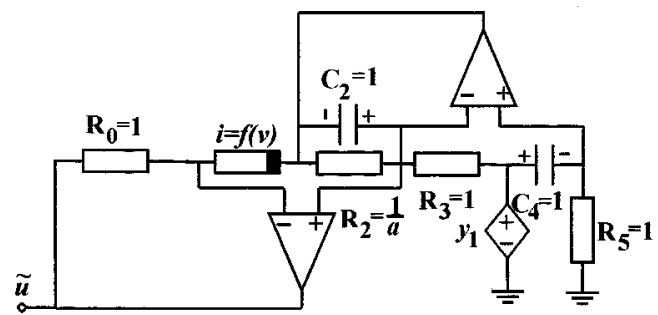

(a)

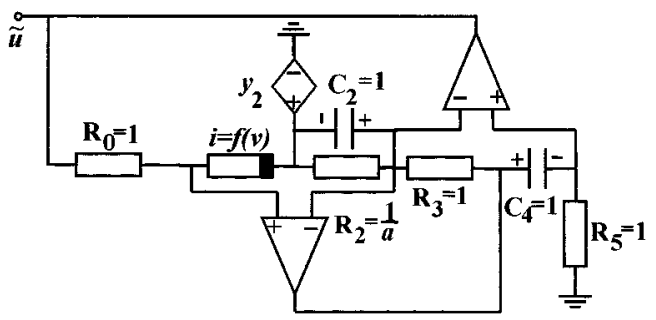

(b)

Figure 8. Two possible inverse systems for the master circuit of Figure 7: (a) the case corresponding to the transmitted variable $y_{1}$; (b) the case corresponding to the transmitted variable $y_{2}$ 
for the circuit of Figure 8(b)

$$
\begin{aligned}
\dot{\tilde{x}}_{1} & =-a \tilde{x}_{1}+\tilde{x}_{2} \\
\dot{\tilde{x}}_{2} & =\tilde{x}_{1}+y_{2} \\
\tilde{u} & =\tilde{x}_{1}+f\left(\tilde{x}_{1}\right)+y_{2}
\end{aligned}
$$

The synchronization of the receivers results from the analysis of the non-autonomous dynamical systems described by the first two equations of (15) and (16). These equations are linear. While the first configuration is stable (possessing two negative eigenvalues equal to -1 and $-a$ ), the second one is unstable, being characterized by a positive eigenvalue. The simulated phase portrait $x_{1}-x_{2}$ and the waveforms of the master driving signal, $u(t)=q \cos \omega t$, and the output of the slave of Figure 8(a), $\tilde{u}(t)$, are depicted in Figure 9. There have been used the following normalized parameters: $a=0 \cdot 4, p=-1 \cdot 1, q=1 \cdot 8, \omega=1 \cdot 8$ that ensures the chaotic behaviour of Duffing equation. ${ }^{14}$ In simulations these parameters have been denormalized using a normalizing resistance of $1 \mathrm{k} \Omega$ and a normalizing frequency of $10 \mathrm{kHz}$ and there have been used 741-type op-amps.

\subsection{Example 2: Chua's circuit}

In the following a communication scheme using Chua's circuit ${ }^{15}$ will be implemented using the proposed method. Chua's circuit has been realized in a variety of ways, both in discrete and integrated implementations, using different electronic devices and components. One of these implementations, reported as a very robust one, has been proposed in Reference 16. In this realization the well-known Chua diode has been obtained using a parallel connection of two negative resistance convertor (NRC) configurations. The current-voltage characteristic of the resulting non-linear resistor is depicted in Figure 10. However, the region of interest for the chaotic behaviour of Chua's circuit is between $-B_{1}$ and $B_{1}$, that is one of the two op-amps does not saturate and it functions always in the linear region. This op-amp can be modelled by a nullor. Its output voltage can be used as the transmitted variable towards a slave circuit which can be built using the above-described procedure. If the information signal $m(t)$ is introduced in the chaotic emitter circuit in the same manner as it is proposed in Reference 17 the structure of Figure 11 results. This circuit is described by the state equation

$$
\begin{aligned}
C_{1} \frac{\mathrm{d} v_{C_{1}}}{\mathrm{~d} t} & =G\left(v_{C_{2}}-v_{C_{1}}\right)-f\left(v_{C_{1}}+m(t)\right) \\
C_{2} \frac{\mathrm{d} v_{C_{2}}}{\mathrm{~d} t} & =G\left(v_{C_{1}}-v_{C_{2}}\right)+i_{L} \\
L \frac{d i_{L}}{\mathrm{~d} t} & =-v_{C_{2}}
\end{aligned}
$$

and the transmitted signal $y(t)$ (the output voltage of $\mathrm{A}_{1}$ ) is given by

$$
y(t)=\left(1+\frac{R_{21}}{R_{31}}\right)\left(v_{C_{1}}(t)+m(t)\right)
$$

Representing $\mathrm{A}_{1}$ as a nullator-norator pair (since $\mathrm{A}_{1}$ is functioning only in the linear region) and using the proposed method for realizing the inverse system, the structure of Figure 12 is obtained:

$$
\begin{aligned}
C_{1} \frac{\mathrm{d} \tilde{v}_{C_{1}}}{\mathrm{~d} t} & =G\left(\tilde{v}_{C_{2}}-\tilde{v}_{C_{1}}\right)-i \\
C_{2} \frac{\mathrm{d} \tilde{v}_{C_{2}}}{\mathrm{~d} t} & =G\left(\tilde{v}_{C_{1}}-\tilde{v}_{C_{2}}\right)+\tilde{i}_{L} \\
L \frac{\mathrm{d} \tilde{i}_{L}}{\mathrm{~d} t} & =-\tilde{v}_{C_{2}}
\end{aligned}
$$




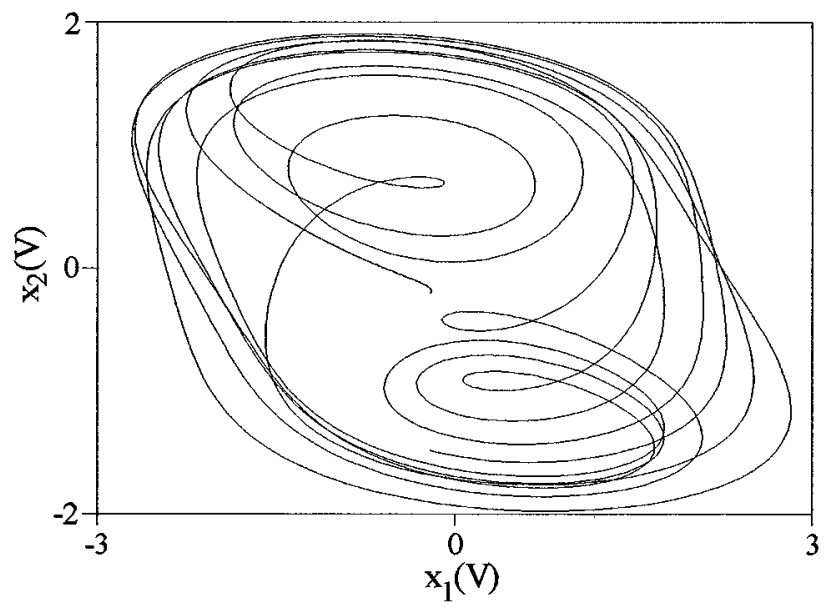

(a)

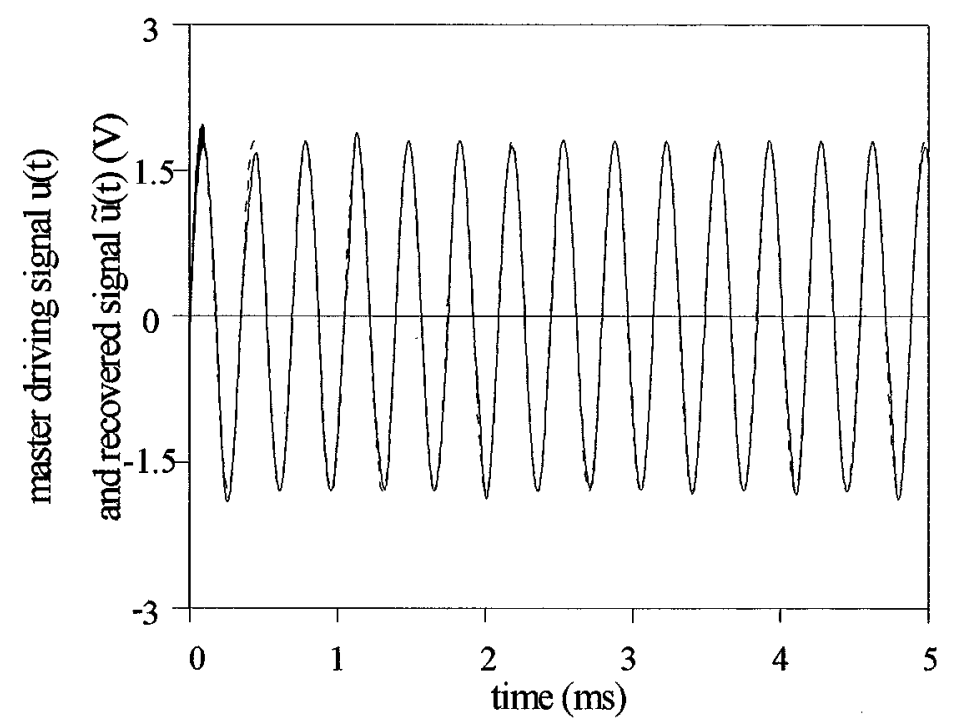

(b)

Figure 9. Simulation results for the chaos synchronization of Duffing equation: (a) master's phase portrait; (b) master driving signal $u$ (dotted lines) and recovered signal $\tilde{u}$ (continuous lines)

where

$$
i(t)=\frac{R_{21}}{\left(R_{21}+R_{31}\right) R_{11}} y(t)-f_{2}\left(\frac{R_{31}}{R_{21}+R_{31}} y(t)\right)
$$

$f_{2}(\cdot)$ describing the driving point $i-v$ characteristic of the non-linear resistor $\mathrm{NR}_{2}$ realized with the op-amp $\mathrm{A}_{2}$. The recovered signal is given by

$$
\tilde{m}(t)=\frac{R_{31}}{R_{21}+R_{31}} y(t)-\tilde{v}_{C_{1}}(t)
$$



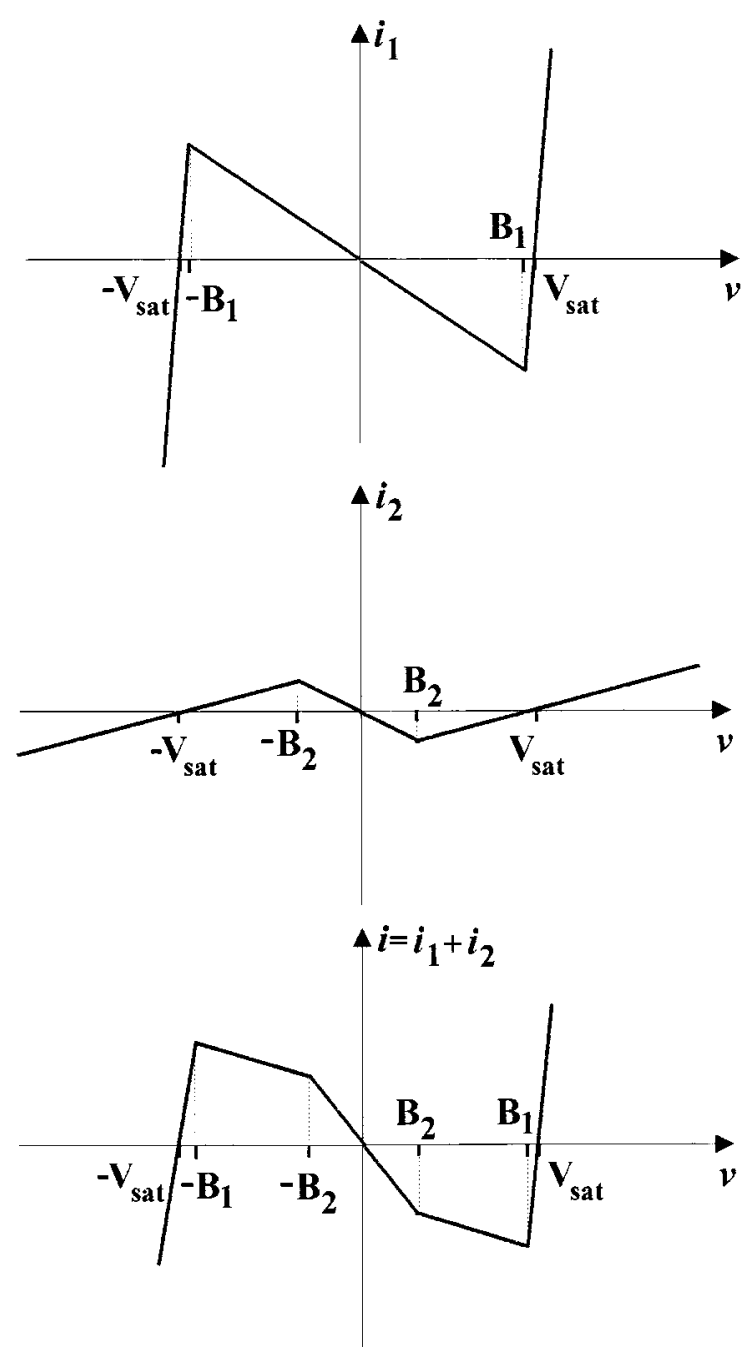

Figure 10. Implementation of Chua's diode by a parallel connection of two NRC circuits

If $\tilde{v}_{C_{1}} \rightarrow v_{C_{1}}$ as $t \rightarrow \infty$ then $\tilde{m}(t) \rightarrow m(t)$. The SPICE simulated waveforms of the transmitted signal $y(t)$, information signal $m(t)$ and recovered signal $\tilde{m}(t)$ are given in Figure 13. The following parameters have been used for the transmitter (from Reference 16): $C_{1}=10 \mathrm{nF}, C_{2}=100 \mathrm{nF}, L=18 \mathrm{mH}, R=1800 \Omega$, $R_{11}=R_{21}=220 \Omega, R_{31}=2200 \Omega, R_{12}=R_{22}=22 \mathrm{k} \Omega, R_{32}=3300 \Omega$ and 741-type op-amps biased with $\pm 9 \mathrm{~V}$ supply voltages. The frequency-dependent finite gain of op-amp $\mathrm{A}_{1}$ has a parasitic influence over the synchronization process. The analysis of the master-slave configuration, taken into account the one-pole model for the op-amps, has shown that the effect of the frequency-dependent gain of $\mathrm{A}_{1}$ can be drastically diminished by increasing at the receiver the value of $C_{1}$ with the quantity

$$
\Delta C_{1}=\frac{\left(G_{21}+G_{31}\right)\left(G+G_{11}+G_{12}\right)}{G_{21} \omega_{t}}
$$

where $\omega_{t}\left(=2 \pi \times 10^{6}\right)$ is the gain-bandwidth product of op-amp $\mathrm{A}_{1}$. With the above values of components it results $\Delta C_{1}=0.9 \mathrm{nF}$. In Figure 13 both waveforms of the recovered signal corresponding to $C_{1}=10 \mathrm{nF}$ and $C_{1}=10.9 \mathrm{nF}$ are depicted. 


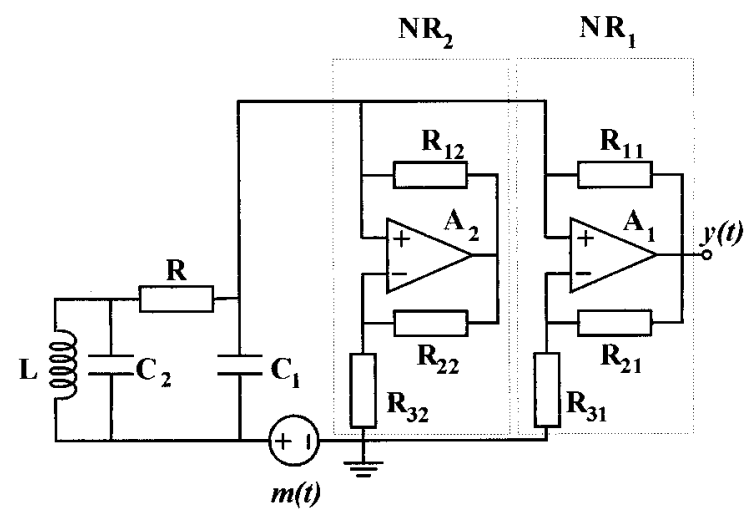

Figure 11. Transmitter from communication scheme using chaos reported in Reference 17 and based on the realization of Chua's circuit proposed in Reference 16

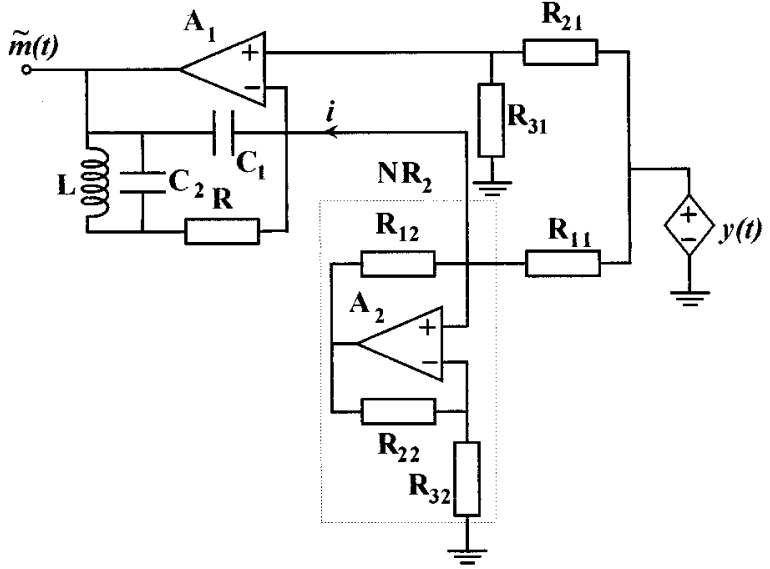

Figure 12. Inverse system of master circuit of Figure 11

\section{NULLOR-BASED METHOD FOR REALIZING INVERSE SYSTEMS FOR MASTER CIRCUITS WITHOUT NULLORS}

Besides the nullor-based implementations of the chaotic circuits there are numerous realizations that do not use electronic devices modelled by nullors. In this section a method for building an inverse system that uses a similar structure with the master one's and a supplementary nullor is introduced. This method can be used no matter the nature of the transmitted variable is: current or voltage.

We consider a nonautonomous chaotic circuit and want to use it in a synchronization scheme based on the inverse system approach. If the transmitted variable is a voltage, one can always augment the master circuit with a series connection nullator-norator between the two nodes across which the transmitted voltage is taken (Figure 14(a)). Since the series connection nullator-norator behaves like an open circuit, it does not affect the functioning of the chaotic circuit, and the voltage across the norator is equal to the output voltage. The inverse system can be built starting from this modified configuration of the master and using the procedure described in the previous section. This is illustrated in Figure 14 in the case of a master system driven by a voltage source, but similar results can be derived in the case of current driving source.

If the transmitted variable is a current, one can modify the master circuit by introducing a parallel connection nullator-norator in series with the branch through which flows the variable to be transmitted (Figure 15(a)). The nullator-norator parallel configuration behaves like a short-circuit and the current through the norator is equal to the output current. The inverse system can be realized by the same procedure as above (Figure 15(b)).

\section{CONCLUSIONS}

A straightforward and simple technique to implement the inverse system for a master non-autonomous circuit is proposed. The method makes use of nullors, known to be the most important active circuit elements. In the process of implementing the inverse system one could use the nullors already existent in the master circuit or a supplementary nullor can be added in the slave structure. The main application of the proposed synthesis method is in chaos synchronization of non-autonomous non-linear circuits and as well as in communications using chaos. A major condition which has to be fulfilled in order to ensure the synchronization is the receiver unique asymptotic behaviour. This depends not only on the transmitted variable (which sets the slave structure), but also on the implementation of nullors. As it is proved in 


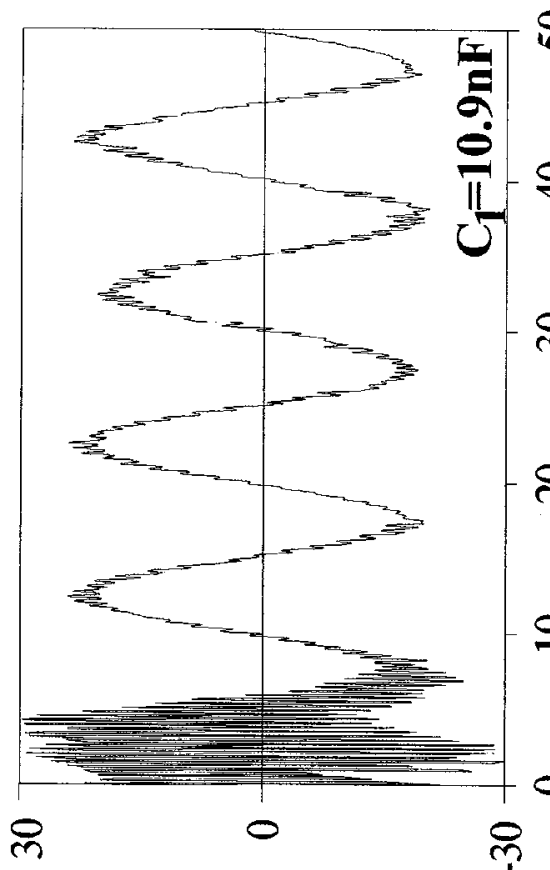

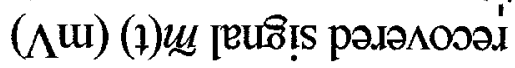
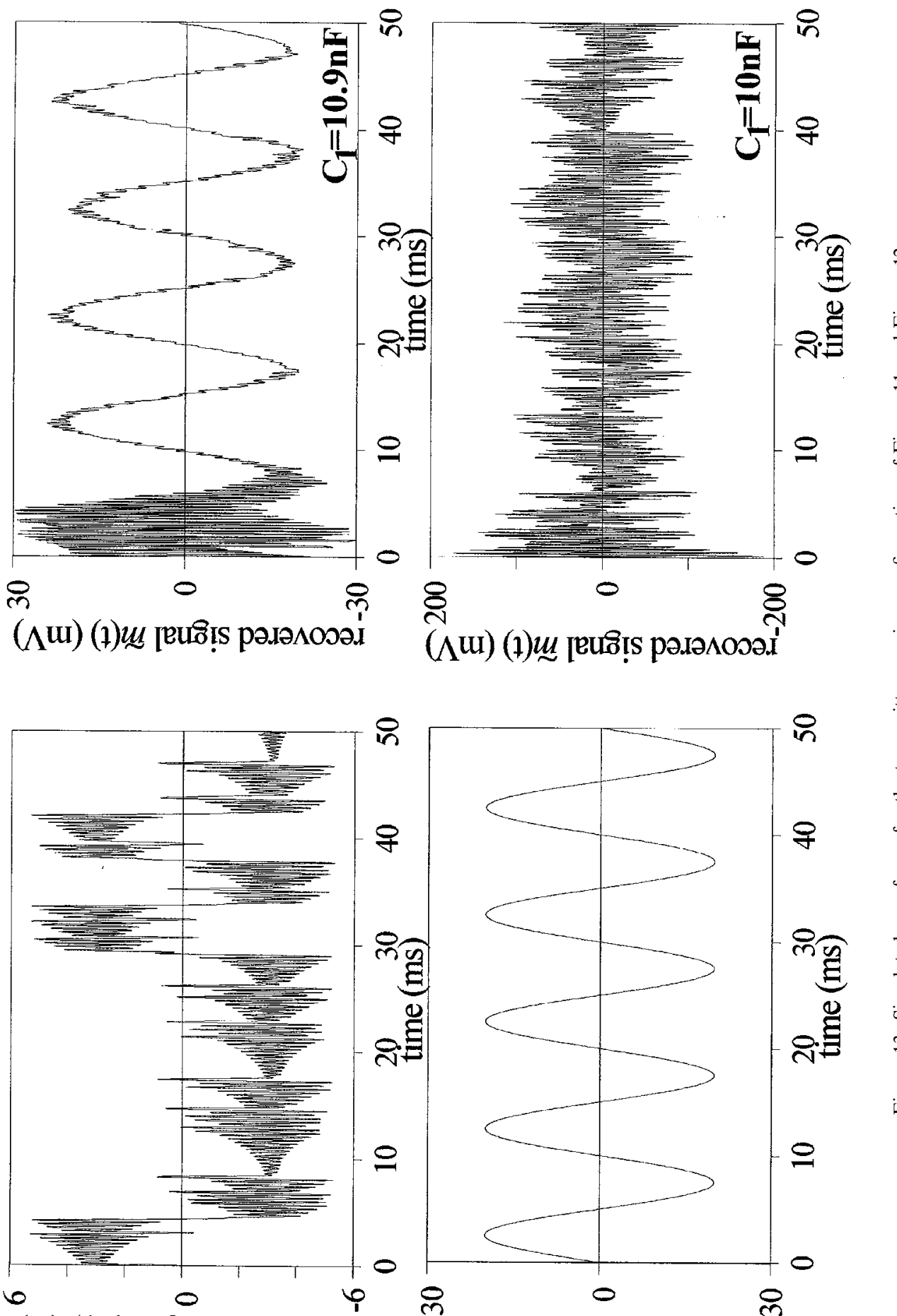

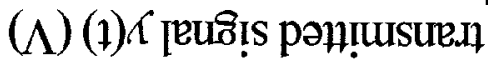

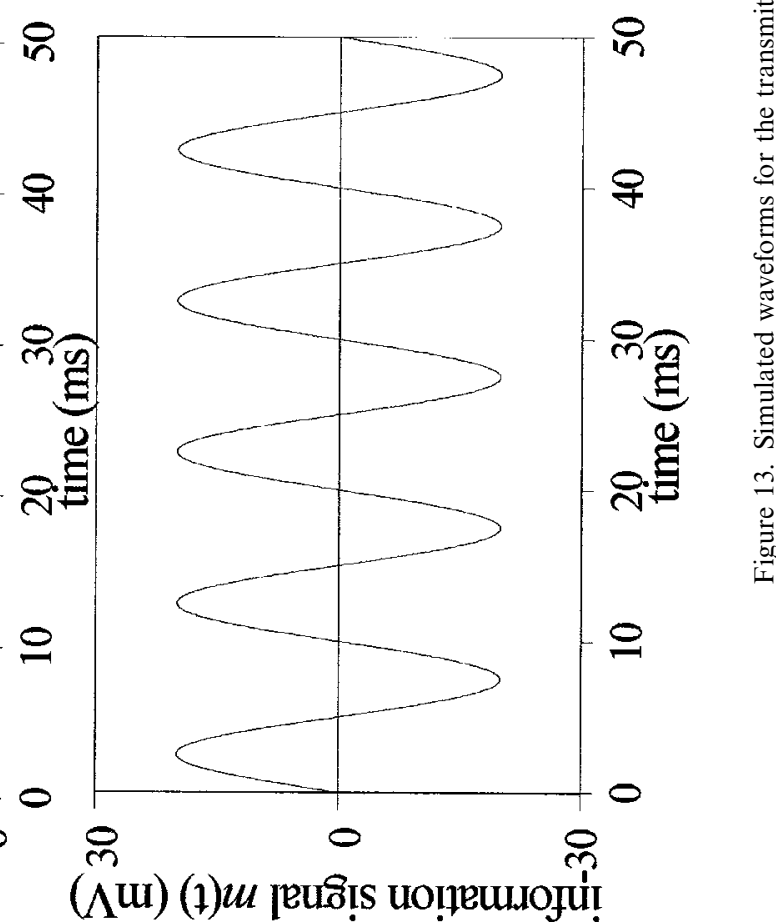




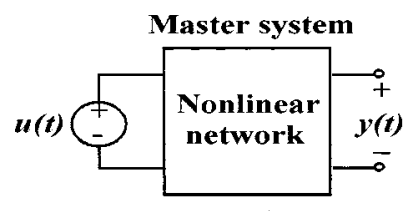

(a)

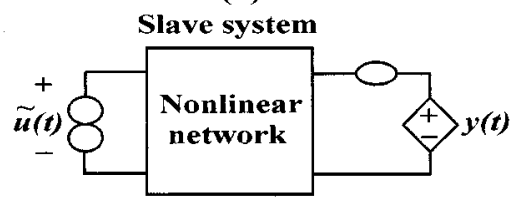

(b)

Figure 14. Nullor-based inverse system realization for nonlinear circuits without nullors in the case of voltage output: (a) the master circuit; (b) inverse system realization

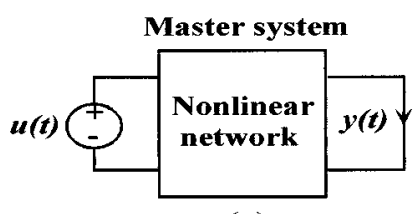

(a)

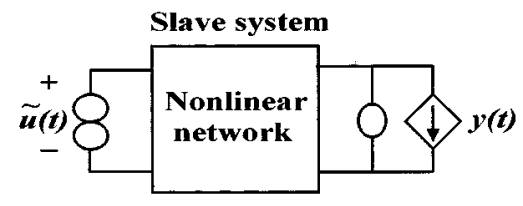

(b)

Figure 15. Nullor-based inverse system realization for non-linear circuits without nullors in the case of current output: (a) the master circuit; (b) inverse system realization

Reference 18 a nullor can be viewed as a limit case (infinite gain) of any class of floating controlled sources (VCVS, VCCS, CCVS, CCCS). Since in practice and simulations one has to use finite gain-controlled sources, this can lead to important errors in the synchronization process or even to instability of the receiver. ${ }^{19}$ The frequency-dependent gain of the controlled sources can produce further errors in the synchronization process, as shown in Example 2. These errors, caused by non-ideal implementation of nullors, have to be analysed carefully in order to achieve synchronization.

\section{ACKNOWLEDGEMENT}

The author wants to thank Professor Liviu Goras and anonymous reviewers for their useful comments and suggestions.

\section{REFERENCES}

1. L. M. Pecora and T. L. Carroll, 'Synchronization in chaotic systems', Phys. Rev. Lett., 64, 821-824 (1990).

2. M. Hasler, 'Synchronization principles and applications', Circuits and Systems Tutorials, ISCAS'94, London, 1994, pp. 314-327.

3. T. L. Carroll, 'Communicating by use of filtered, synchronized, chaotic signals', IEEE Trans. Circuit System I, CAS-42, 105-110 (1995).

4. M. J. Ogorzalek, 'Control concepts and applications', Circuits and Systems Tutorials, ISCAS' 94, London, 1994, pp. 328-340.

5. M. J. Ogorzalek, 'Taming chaos - Part I: Synchronization', IEEE Trans. Circuit Systems I, CAS-40, 693-699 (1993).

6. M. J. Ogorzalek, 'Taming chaos-Part II: Control', IEEE Trans. Circuit Systems I, CAS-40, 700-706 (1993).

7. U. Feldmann, M. Hasler and W. Schwarz, 'Communication by chaotic signals: the inverse system approach', Int. J. Circuit Theory Appl., 24, 551-579 (1996).

8. F. Böhme and W. Schwarz, 'The chaotizer-dechaotizer-channel', IEEE Trans. Circuit Systems I, CAS-43, $569-599$ (1996).

9. T. L. Carroll and L. M. Pecora, 'Synchronizing chaotic circuits', IEEE Trans. Circuit Systems, CAS-38, 453-456 (1991).

10. J. Malhotra and R. Senani, 'Class of floating, generalised, positive/negative immittance convertors/inverters realised with operational mirrored amplifiers', Electronic Lett., 30, 3-5 (1994).

11. C. Toumazou and F. J. Lidgey, Analog IC Design: the Current-Mode Approach, Peter Peregrinus Ltd., London, 1990.

12. L. Goras, 'Linear and nonlinear mutators derived from GIC-type configurations', IEEE Trans. Circuit Systems, CAS-28, 165-168 (1981).

13. L. Goras, A. Leuciuc and I. Ciocoiu, 'On nonlinear synthesis using generalized mutators', Proc. NDES'94, Krakow, Poland, 1994, pp. $179-184$.

14. G. Chen and X. Dong, 'On feedback control of chaotic continuous-time systems', IEEE Trans. Circuit Systems I, CAS-40, 591-601 (1993).

15. T. Matsumoto, L. O. Chua and M. Komuro, 'The double scroll', IEEE Trans. Circuit System, CAS-32, 797-818 (1985).

16. M. P. Kennedy, 'Robust op-amp realization of Chua's circuit', Frequenz 46, 66-80 (1992).

17. C. W. Wu and L. O. Chua, 'A simple way to synchronize chaotic systems with applications to secure communication systems', Int. J. Bifurcation Chaos, 3, 1619-1627 (1993).

18. R. Cabeza, A. Carlosena and L. Serrano, 'Unified approach to the implementation of universal active devices', Electronic Lett., 30 , 618-620 (1994).

19. A. Leuciuc, 'On the effect of nonideal implementation of nullors in chaos synchronization', Proc. NDES'96, Sevilla, Spain, 1996, pp. $183-188$. 